\title{
Rotating deformed halo nuclei and shape decoupling effects
}

\author{
Xiang-Xiang Sun (孙向向) $)^{1,2,3}$ and Shan-Gui Zhou (周善贵) ${ }^{2,3,4,5, *}$ \\ ${ }^{1}$ School of Nuclear Science and Technology, University of Chinese Academy of Sciences, Beijing 100049, China \\ ${ }^{2}$ CAS Key Laboratory of Theoretical Physics, Institute of Theoretical Physics, \\ Chinese Academy of Sciences, Beijing 100190, China \\ ${ }^{3}$ School of Physical Sciences, University of Chinese Academy of Sciences, Beijing 100049, China \\ ${ }^{4}$ Center of Theoretical Nuclear Physics, National Laboratory of Heavy Ion Accelerator, Lanzhou, 730000, China \\ ${ }^{5}$ Synergetic Innovation Center for Quantum Effects and Application, \\ Hunan Normal University, Changsha, 410081, China
}

(Dated: March 24, 2021)

\begin{abstract}
We explore the rotational feature of deformed halos by performing the angular momentum projection (AMP) on the ground state wave functions obtained from the deformed relativistic Hartree-Bogoliubov theory (DRHBc) in continuum. The DRHBc+AMP approach self-consistently describes the coupling between single particle bound states and the continuum not only in the ground state but also in rotational states. The rotational modes of deformed halos in ${ }^{42} \mathrm{Mg}$ and ${ }^{44} \mathrm{Mg}$ are investigated by studying properties of rotational states such as excitation energy, configuration, and density distribution. Our study demonstrates that the deformed halo structure persists from the ground state in the intrinsic frame to collective states. Especially, the typical behavior of shape decoupling effects in rotating deformed halo nuclei is revealed.
\end{abstract}

Many exotic phenomena in atomic nuclei, e.g., the shell evolution, halos, clustering effects, and shape coexistence, are closely related to deformation effects originating from quantum correlations of valence nucleons [1-11]. Nuclear halo, firstly observed in ${ }^{11} \mathrm{Li}$ [12], is characterized by weak binding and large spatial extension due to the considerable occupation of low- $l$ ( $s$ - or $p$-wave) orbitals of valence nucleon(s) close to the threshold of the particle emission [13-19]. In deformed weakly bound nuclei, the breaking of spherical symmetry increases the density of single particle levels (SPLs) around the Fermi surface and the number of SPLs with low- $l$ components, thus contributing to the formation of deformed halos [20, 21], such as those observed in ${ }^{31} \mathrm{Ne}$ $[22,23]$ and ${ }^{37} \mathrm{Mg}$ [24]. Furthermore, the halo structure is also connected with the shell evolution $[25,26]$ and the interplay between deformed halos and shell evolution is particularly complex and interesting [27-32]. It is well established that the intrinsic shape and shell structure manifest themselves in the corresponding low-lying excited spectra. Therefore, studying the collective motion, especially rotational excitations, of deformed halos is helpful for understanding the halo configuration and related exotic structures.

The self-consistent descriptions of ground states of deformed halo nuclei have been achieved with the deformed relativistic Hartree-Bogoliubov theory in continuum (DRHBc) $[21,33,34]$. By using the DRHBc theory, it has been predicted that ${ }^{42,44} \mathrm{Mg}$ are deformed halo nuclei with shape decoupling effects: The core has a prolate shape while the halo is slightly oblate [21, 33]. Such shape decoupling effects are the consequence of the intrinsic structure of valence SPLs [20, 21,33]. More deformed halos have been predicted in ${ }^{15,22} \mathrm{C}[29,35]$, ${ }^{32,34,36,38} \mathrm{Ne}[21,36,37],{ }^{37,40} \mathrm{Mg}[38]$, and even heavier nuclei [39]; some of them exhibit shape decoupling effects. A remarkable question is how the core and halo behave in rotating deformed halo nuclei.

The rotational excitation of deformed nuclei can be studied by using the angular momentum projection (AMP) technique which is one of beyond mean field methods and has been widely applied to the study of exotic nuclear structures [6, 40,41], the shape coexistence [42-45] and shape evolution [46-48], triaxial nuclear shapes [49-55], nuclear chirality [56, 57], and fission and structure of superheavy nuclei $[58,59]$. In this Letter, we investigate the rotational excitation of deformed halo nuclei and the typical behavior of the halo and core in low-lying rotational states by implementing the AMP in the DRHBc theory. To guarantee a proper description of the asymptotic behavior of the wave function in halo nuclei, the projected wave function is expanded in terms of the Dirac Woods-Saxon (WS) basis [60], similar to what has been done for the mean field (MF) wave function in the DRHBc theory.

The details and applications of the DRHBc theory can be found in Refs. $[21,29,33,35,61-66]$. In the DRHBc+AMP approach, a low-lying rotational state $|J M\rangle$ with the angular momentum $J$ and its projection $M$ along $z$ axis in laboratory frame is constructed by performing AMP on the intrinsic wave function $\left|\Phi\left(\beta_{2}\right)\right\rangle$ obtained from the DRHBc calculation with a certain quadrupole deformation parameter $\beta_{2}$

$$
\left|\Psi^{J M}\right\rangle=f^{J} \hat{P}_{M 0}^{J}\left|\Phi\left(\beta_{2}\right)\right\rangle,
$$

with the weight $f^{J}$ and angular momentum projection operator $\hat{P}_{M 0}^{J}$ written in terms of an integral over the Euler angles [67]. The energy $E^{J}$ and weight $f^{J}$ can be obtained by solving the Hill-Wheeler equation [67]. For axially symmetric nuclei the solution is simplified as 
$[68,69]$

$$
\begin{aligned}
& E^{J}=\frac{\left\langle\Phi\left(\beta_{2}\right)\left|\hat{H} \hat{P}_{00}^{J}\right| \Phi\left(\beta_{2}\right)\right\rangle}{\left\langle\Phi\left(\beta_{2}\right)\left|\hat{P}_{00}^{J}\right| \Phi\left(\beta_{2}\right)\right\rangle}, \\
& f^{J}=\frac{1}{\sqrt{\left\langle\Phi\left(\beta_{2}\right)\left|\hat{P}_{00}^{J}\right| \Phi\left(\beta_{2}\right)\right\rangle}} .
\end{aligned}
$$

In the present work we focus on low-lying rotational states with positive parity. The reduced transition probability from an initial state $J_{i}$ to a final state $J_{f}$ is calculated as [70]

$$
B\left(E 2, J_{i} \rightarrow J_{f}\right)=\frac{e^{2}}{2 J_{i}+1}\left|f^{J_{f} *}\left\langle J_{f}\left\|\widehat{Q}_{2}\right\| J_{i}\right\rangle f^{J_{i}}\right|^{2},
$$

and the spectroscopic quadrupole moment for a state with spin $J$ is

$$
Q^{(\mathrm{s})}(J)=e \sqrt{\frac{16 \pi}{5}}\left(\begin{array}{ccc}
J & 2 & J \\
J & 0 & -J
\end{array}\right)\left(f^{J}\right)^{2}\left\langle J\left\|\hat{Q}_{2}\right\| J\right\rangle,
$$

where $\hat{Q_{2}}$ is the electric quadrupole moment operator. In the $r$ space the one-body density of a rotational state is defined as $[71,72]$

$$
\rho^{J}(\boldsymbol{r})=\left\langle\Psi^{J M}\left|\sum_{i} \delta\left(\boldsymbol{r}-\boldsymbol{r}_{i}\right)\right| \Psi^{J M}\right\rangle,
$$

where the index $i$ represents all occupied single particle sates of neutrons and protons.

The Dirac WS basis is obtained by solving the Dirac equation with spherical scalar and vector potentials of the WS form [60] and consists of spherical basis states labelled by $|n l j\rangle$ with the radial quantum number $n$, the orbital angular momentum $l$ of the large component of the Dirac spinor, and the total angular momentum $j$. The configuration of the valence nucleon(s) is obtained by calculating the probability amplitude of spherical components of valence levels. In the DRHBc theory, the probability amplitude of a spherical component is

$$
N_{n l j}^{\mathrm{DRHBc}}=\left\langle\Phi\left(\beta_{2}\right)\left|\hat{N}_{n l j}\right| \Phi\left(\beta_{2}\right)\right\rangle,
$$

where $\hat{N}_{n l j}=\sum_{m} c_{n l j m}^{\dagger} c_{n j l m}$ with $m$ being the projection of total angular momentum on the symmetry axis. For each excited state, the probability amplitude of $|n l j\rangle$ is calculated as [73]

$$
N_{n l j}^{J}=\frac{\left\langle\Phi\left(\beta_{2}\right)\left|\hat{N}_{n l j} \hat{P}_{00}^{J}\right| \Phi\left(\beta_{2}\right)\right\rangle}{\left\langle\Phi\left(\beta_{2}\right)\left|\hat{P}_{00}^{J}\right| \Phi\left(\beta_{2}\right)\right\rangle} .
$$

In this work, the density functional PC-PK1 [74] and a density-dependent $\delta$ force with the strength of $342.5 \mathrm{MeV}$ $\mathrm{fm}^{3}$ [65] are adopted in the particle-hole and particleparticle channels respectively. The box size used to generate the Dirac WS basis is $R_{\text {box }}=20 \mathrm{fm}$. The energy truncation for positive energy states of the Dirac WS basis is $E_{\text {cut }}^{+}=300 \mathrm{MeV}$ and the number of basis states in the Dirac sea equals that in the Fermi sea.

Next we study in detail the rotational behaviors of magnesium isotopes close to the neutron drip line which has not been well defined yet. ${ }^{40} \mathrm{Mg}$ is the heaviest magnesium isotope observed so far [75] and the recently established low-lying excited spectrum [76] indicates that the shell closure at $N=28$ is broken down. With Monto Carlo Shell Model (MCSM) calculations, it is revealed that ${ }^{42} \mathrm{Mg}$ is a dripline nucleus as a consequence of deformation [77]. ${ }^{44} \mathrm{Mg}$ is possibly bound from the very recent $a b$ initio prediction [78]. Several self-consistent MF calculations $[33,79,80]$ have predicted that the neutron dripline is located at $N=32 \pm 2$ for $\mathrm{Mg}$ isotopes. In the DRHBc prediction with PC-PK1 [62, 65], this isotopic chain ends at $N=34$. Especially, ${ }^{42} \mathrm{Mg}$ and ${ }^{44} \mathrm{Mg}$ are predicted to be deformed two-neutron $(2 n)$ and four-neutron $(4 n)$ halo nuclei with shape decoupling effects $[21,33]$ because the configuration of valence neutrons has a significant $p$-wave amplitude. ${ }^{40,42} \mathrm{Mg}$ and ${ }^{44} \mathrm{Mg}$ offer prototype systems to explore how the deformation influences the shell evolution, halo configuration, and rotational excitation of weakly bound nuclei.

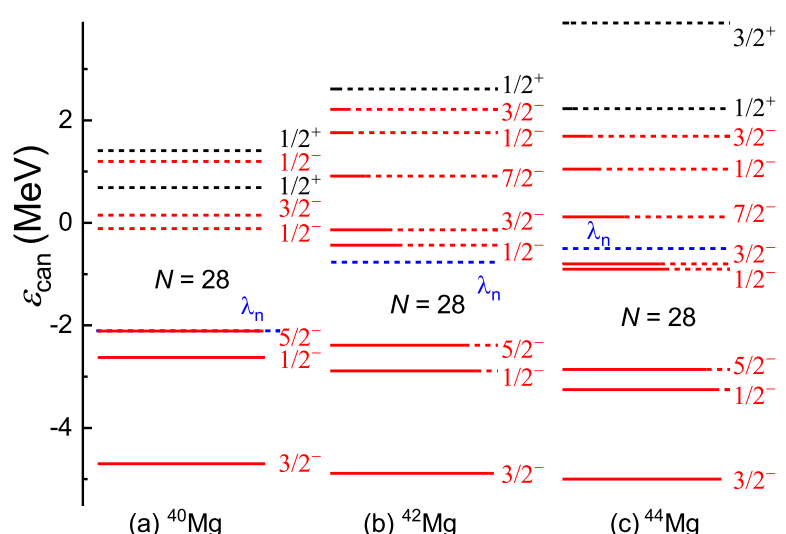

FIG. 1. Single neutron levels around the Fermi surface $\left(\lambda_{n}\right)$ of ${ }^{40,42,44} \mathrm{Mg}$ in the canonical basis. The length of the solid line is proportional to the occupation probability $v^{2}$ of each level labelled by $m^{\pi}$ where $\pi$ is the parity. Levels with negative and positive parities are presented by red and black lines.

In DRHBc calculations with PC-PK1, ${ }^{40} \mathrm{Mg},{ }^{42} \mathrm{Mg}$, and ${ }^{44} \mathrm{Mg}$ are well deformed with $\beta_{2}=0.46,0.38$, and 0.31 respectively. Figure 1 shows the single neutron levels around the Fermi surface in the canonical basis for them. It is found that for ${ }^{40,42,44} \mathrm{Mg}$ the energy gaps at $N=28$ are about $2 \mathrm{MeV}$, which is much smaller than the spherical shell gap at $N=28$ in the shell model [67] and indicates that the shell closure at $N=28$ is quenched 


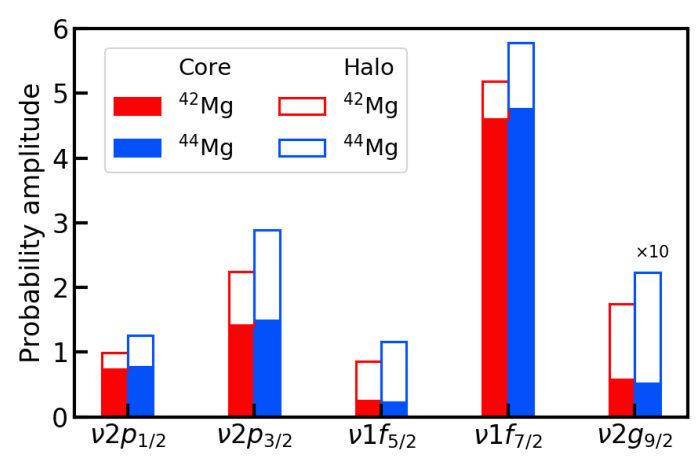

FIG. 2. Probability amplitude of $p$-, $f$-, and $g$-wave components for neutrons $(\nu)$ contributed to the core and halo of ${ }^{42,44} \mathrm{Mg}$ from DRHBc calculations. The probability amplitude of $\nu 2 g_{9 / 2}$ is multiplied by 10 .

due to deformation effects in the MF level. The valence orbital of ${ }^{40} \mathrm{Mg}$ is the level $5 / 2^{-}$dominated by $f$-wave and the level closest to it is $1 / 2^{-}$which contains $p$-wave component with a small amplitude. Thus the ground state of ${ }^{40} \mathrm{Mg}$ does not show a halo structure, which is opposite to the conclusion given in Ref. [38]. For halo nuclei ${ }^{42,44} \mathrm{Mg}$, although small, the gap at $N=28$ can still be used to identify the core and halo: The neutron levels above the gap are valence levels and contribute to the halo and those below it form the core. According to Eq. (6), the probability amplitudes of main spherical components of SPLs in the core and halo are obtained and presented in Fig. 2. For ${ }^{42,44} \mathrm{Mg}$, valence neutrons are mainly dominated by $p$-, $f$ - and $g$-waves and the amplitude of $g$-wave is small. The formation of the halo is due to the appearance of the $p$-wave in valence levels. The densities of the whole nucleus, all neutrons, neutron core and neutron halo in the $x z$ plane are shown in the first row of Fig. 3 for ${ }^{42} \mathrm{Mg}$. It can be seen that the core has a prolate shape while the halo is slightly oblate, which means that ${ }^{42} \mathrm{Mg}$ is a deformed halo nucleus with shape decoupling effects. Similar results can be obtained for ${ }^{44} \mathrm{Mg}$. These conclusions are consistent with the previous predictions given in Refs. [21, 33].

The low-lying excited spectra of ${ }^{40,42,44} \mathrm{Mg}$ are obtained by performing AMP based on the ground state from DRHBc calculations. $B\left(E 2,0^{+} \rightarrow 2^{+}\right)$, the excitation energy $E\left(J^{+}\right)$, and $Q^{(\mathrm{s})}\left(J^{+}\right)$for each collective state are listed in Table I. The calculated excitation energies of $2^{+}$and $4^{+}$of ${ }^{40} \mathrm{Mg}$ are very close to those shown in Refs. [81, 82] with PC-F1 [83], slightly smaller than those calculated results with Gongy force $[70,84]$, and similar to the recent MCSM calculation results [77]. The excitation energy of the $2^{+}$state of ${ }^{40} \mathrm{Mg}$ is $0.54 \mathrm{MeV}$, which agrees with the experimental value given in Ref. [76]. For halo nuclei ${ }^{42} \mathrm{Mg}$ and ${ }^{44} \mathrm{Mg}$, there are no experimental low-lying excited spectra to

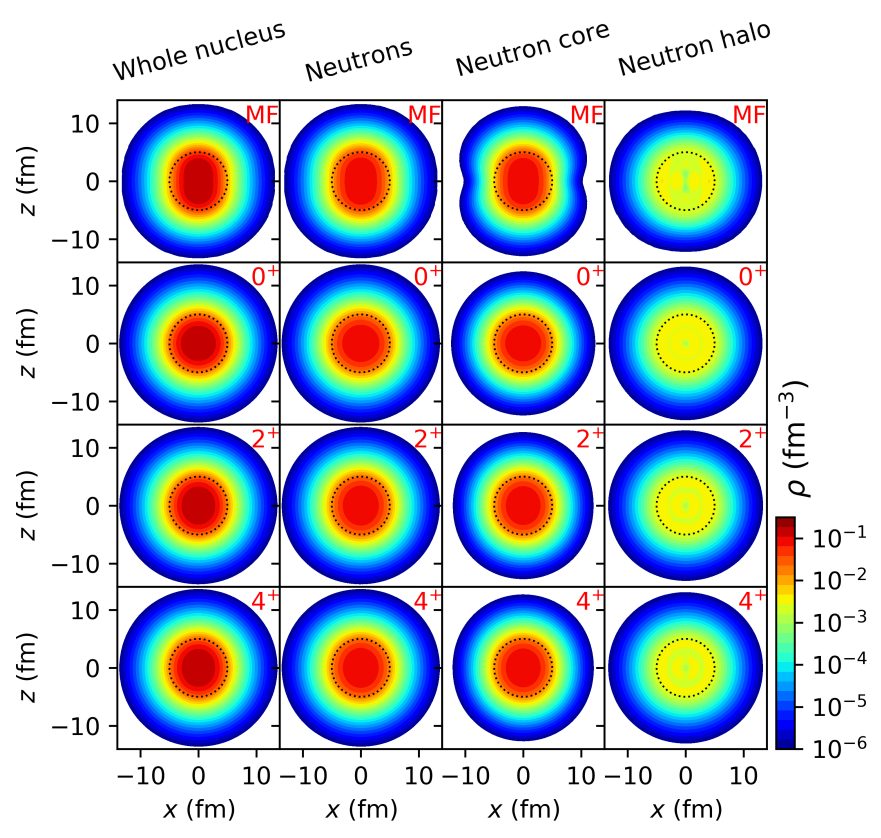

FIG. 3. Densities for the whole nucleus, all neutrons, the neutron core and neutron halo of ${ }^{42} \mathrm{Mg}$ in the MF ground state and angular momentum projected $0^{+}, 2^{+}$, and $4^{+}$states (with $M=0)$. Black dotted circles are given to guide the eye.

date and the calculated values of $E\left(2^{+}\right)$are about 1.40 $\mathrm{MeV}$, which is close to the shell model calculation [85] and much higher than that of ${ }^{40} \mathrm{Mg}$.

TABLE I. Calculated $E\left(J^{+}\right), Q^{(s)}\left(J^{+}\right)$, and $B\left(E 2,0^{+} \rightarrow 2^{+}\right)$ for ${ }^{40,42,44} \mathrm{Mg}$ by using DRHBc+AMP with PC-PK1.

\begin{tabular}{lrrr}
\hline \hline & ${ }^{40} \mathrm{Mg}$ & ${ }^{42} \mathrm{Mg}$ & ${ }^{44} \mathrm{Mg}$ \\
\hline$E\left(2^{+}\right)(\mathrm{MeV})$ & 0.54 & 1.36 & 1.37 \\
$E\left(4^{+}\right)(\mathrm{MeV})$ & 1.86 & 4.51 & 4.90 \\
$E\left(6^{+}\right)(\mathrm{MeV})$ & 4.02 & 8.85 & 9.75 \\
$Q^{(\mathrm{s})}\left(2^{+}\right)\left(e \mathrm{fm}^{2}\right)$ & -18.71 & -18.35 & -17.68 \\
$Q^{(\mathrm{s})}\left(4^{+}\right)\left(e \mathrm{fm}^{2}\right)$ & -23.91 & -23.36 & -22.41 \\
$Q^{(\mathrm{s})}\left(6^{+}\right)\left(e \mathrm{fm}^{2}\right)$ & -26.47 & -25.81 & -24.69 \\
$B\left(E 2,0^{+} \rightarrow 2^{+}\right)\left(e^{2} \mathrm{fm}^{4}\right)$ & 426.71 & 410.03 & 384.47 \\
\hline \hline
\end{tabular}

A typical feature of a rotational band is that the excitation energies have a linear relation with $J(J+1) \hbar^{2}$, i.e., $E\left(J^{+}\right)=\left\langle\hat{J}^{2}\right\rangle / 2 \mathcal{J}$ where $\mathcal{J}$ is the moment of inertia (MoI). Therefore, we fit the calculated excitation energies to this linear relation. The calculated excitation energies of ${ }^{40,42,44} \mathrm{Mg}$ and fitted lines are displayed in Fig. 4. The distinct linear relation indicates that these three nuclei are all good rotors. The MoI of ${ }^{42} \mathrm{Mg}\left(2.34 \mathrm{MeV}^{-1} \hbar^{2}\right)$ is close to that of ${ }^{44} \mathrm{Mg}\left(2.13 \mathrm{MeV}^{-1} \hbar^{2}\right)$ and much smaller than that of ${ }^{40} \mathrm{Mg}\left(5.19 \mathrm{MeV}^{-1} \hbar^{2}\right)$. This can be understood from two aspects. On one hand, if a nucleus is treated as a rigid body, the MoI is proportional to $\beta_{2}$. The value of $\beta_{2}$ for ${ }^{40} \mathrm{Mg}$ is larger that those for ${ }^{42} \mathrm{Mg}$ 
and ${ }^{44} \mathrm{Mg}$ from DRHBc calculations. On the other hand, from Fig. 1, it is seen that pairing correlations play a vital role in ${ }^{42,44} \mathrm{Mg}$ but not in ${ }^{40} \mathrm{Mg}$. Therefore, the MoI of ${ }^{40} \mathrm{Mg}$ is much lager than those of ${ }^{42,44} \mathrm{Mg}$.

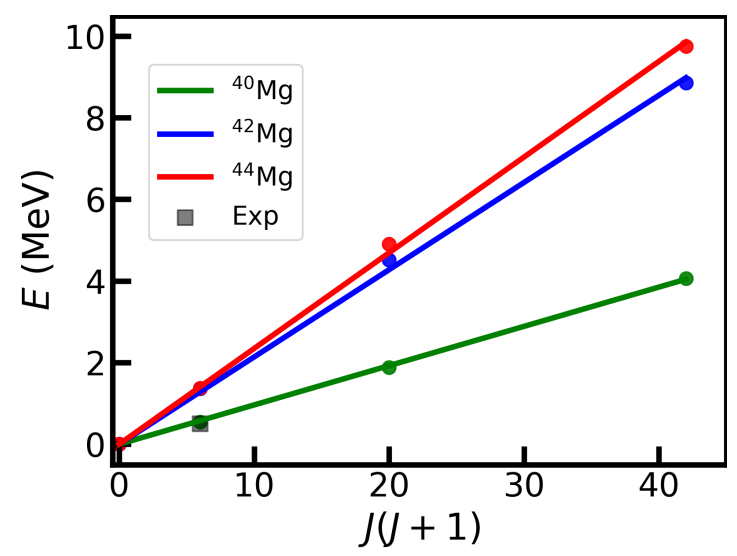

FIG. 4. Excitation energies of collective states as a function of $J(J+1)$ for ${ }^{40,42,44} \mathrm{Mg}$. The calculated results are labelled by solid dots and the linear fitting of calculated spectrum of each nucleus is shown by solid lines. The experimental result of ${ }^{40} \mathrm{Mg}$ taken from Ref. [76] is shown for comparison.

The calculated spectroscopic quadrupole moments of the $2^{+}$and $4^{+}$states for ${ }^{40,42,44} \mathrm{Mg}$ are negative, meaning that the density distribution of $2^{+}\left(4^{+}\right)$state has a prolate shape. Taking ${ }^{42} \mathrm{Mg}$ as an example, the densities for the whole nucleus and for all neutrons, which are displayed in the first and second columns of Fig. 3, have prolate shapes in $2^{+}$and $4^{+}$states. Since the densities in collective states are the expectation value of the onebody operator with respect to $\left|\Psi^{J M}\right\rangle$, the strategy to distinguish the core and halo in the MF level can be applied to collective states. Thus obtained densities for the core and halo in collective states are shown in Fig. 3. Shape decoupling effects of deformed halo in rotational excited states can be studied by examining the shape of density distribution. It is found that the halo still exists in rotational excited states. The densities of the whole nucleus, all neutrons, the neutron core and neutron halo of the $0^{+}$state are all spherical, meaning that shape decoupling effects do not appear due to the nature of spherical symmetry of this state. In the $2^{+}$and $4^{+}$ states, the core has a prolate shape while the halo is slightly oblate. This can be verified by checking the mass quardupole moment of neutrons $Q_{2}^{(n)}$. The $Q_{2}^{(n)}$ values of the core are $-46.47 \mathrm{fm}^{2}$ and $-60.58 \mathrm{fm}^{2}$ for $2^{+}$and $4^{+}$ states respectively while those of the halo are $2.24 \mathrm{fm}^{2}$ and $5.78 \mathrm{fm}^{2}$. The calculated values of $Q_{2}^{(n)}$ are negative for the core but positive for the halo, which is consistent with the prolate shape for the core and oblate shape for the halo, meaning shape decoupling effects in the $2^{+}$and $4^{+}$states.

The halo structure and shape decoupling effects are

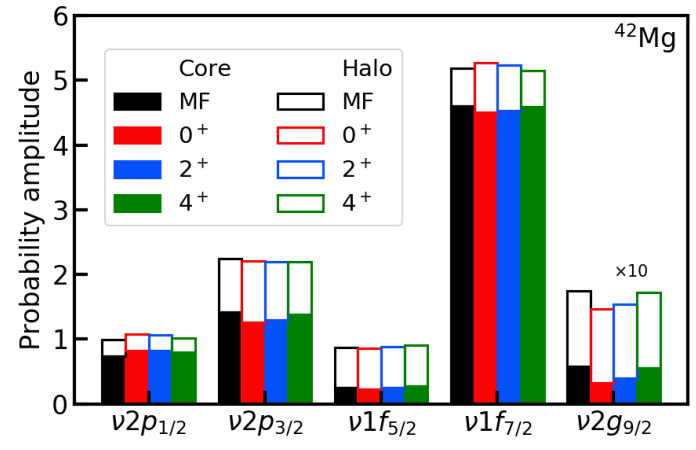

FIG. 5. Probability amplitude of $p$-, $f$-, and $g$-wave components contributed to the halo and core of the MF ground state and each collective state for ${ }^{42} \mathrm{Mg}$. The probability amplitude of $\nu 2 g_{9 / 2}$ is multiplied by 10 .

determined by intrinsic properties of valence levels [21, 29, 33]. To understand the halo configuration and behavior of shape decoupling effects in collective states of ${ }^{42} \mathrm{Mg}$, we calculate probability amplitudes of main spherical components in the neutron core and halo according to Eq. (7), which are displayed in Fig. 5. The valence neutrons are still partially dominated by $p$-wave with a considerable amplitude, resulting in the existence of the halo structure in collective states. Probability amplitudes of $2 p_{1 / 2}$ and $1 f_{5 / 2}$ in collective states almost keep unchanged compared with those of the MF ground state, while the amplitudes for $2 p_{3 / 2}$ and $1 f_{7 / 2}$ decrease slightly with the increase of $J$. The probability amplitudes of $p$-, $f$-, and $g$-waves contributed to the neutron core also change slightly from the MF ground state to collective states. This means that for the rotational excitation of deformed halo nuclei, the configuration of each low-lying rotational states is almost the same as that of the MF ground state. The intrinsic structure of SPLs remains stable from the ground state in the intrinsic frame to the low-lying rotational states. Therefore, the halo structure persists from the ground state in the intrinsic frame to collective states and shape decoupling effects appear in $2^{+}$and $4^{+}$states. As for ${ }^{44} \mathrm{Mg}$ with a $4 n$ halo, the conclusions on the halo structure and shape decoupling effects are similar to ${ }^{42} \mathrm{Mg}$ and we will not discuss it in detail.

In summary, the AMP has been implemented in the DRHBc theory and this newly developed DRHBc+AMP approach is used to study low-lying rotational states of deformed halo nuclei. The rotational bands of deformed $2 n$ halo nucleus ${ }^{42} \mathrm{Mg}$ and $4 n$ halo nucleus ${ }^{44} \mathrm{Mg}$ are obtained by performing AMP on the ground state wave functions obtained from DRHBc calculations with PCPK1. By calculating the probability amplitudes of main spherical orbital components of valence levels, it is found that the configuration of valence neutrons 
changes slightly from the MF ground state to collective states. Therefore the halo structure persists from the ground state in the intrinsic frame to rotational excited states. As for shape decoupling effects of deformed halos, our study demonstrates that this exotic structure does not appear in the $0^{+}$state but exists in the $2^{+}$ and $4^{+}$states. From the structure of SPLs in the MF level and calculated low-lying excited spectrum, it is found that the shell closure at $N=28$ is quenched due to deformation effects, which is also essential for determining the configurations of deformed halos in the intrinsic and collective states. Therefore the information on low-lying excited spectra is particularly important for revealing the deformed halo structure.

We thank the DRHBc Mass Table Collaboration for helpful discussions. This work has been supported by the National Key R\&D Program of China (Grant No. 2018YFA0404402), the National Natural Science Foundation of China (Grants No. 11525524, No. 12070131001, No. 12047503, and No. 11961141004), the Key Research Program of Frontier Sciences of Chinese Academy of Sciences (Grant No. QYZDB-SSWSYS013), the Strategic Priority Research Program of Chinese Academy of Sciences (Grant No. XDB34010000), the Inter-Governmental S\&T Cooperation Project between China and Croatia, and the IAEA Coordinated Research Project "F41033". The results described in this paper are obtained on the High-performance Computing Cluster of ITP-CAS and the ScGrid of the Supercomputing Center, Computer Network Information Center of Chinese Academy of Sciences.

* sgzhou@itp.ac.cn

[1] M. Bender, P.-H. Heenen, and P.-G. Reinhard, Rev. Mod. Phys. 75, 121 (2003).

[2] S. Ćwiok, P.-H. Heenen, and W. Nazarewicz, Nature 433, 705 (2005).

[3] J. Meng, H. Toki, S. Zhou, S. Zhang, W. Long, and L. Geng, Prog. Part. Nucl. Phys. 57, 470 (2006).

[4] K. Heyde and J. L. Wood, Rev. Mod. Phys. 83, 1467 (2011).

[5] J. Meng and S.-G. Zhou, J. Phys. G: Nucl. Part. Phys. 42, 093101 (2015).

[6] T. Nikšić, D. Vretenar, and P. Ring, Prog. Part. Nucl. Phys. 66, 519 (2011).

[7] J. Meng, ed., Relativistic Density Functional for Nuclear Structure (World Scientific, 2016).

[8] S.-G. Zhou, Phys. Scr. 91, 063008 (2016).

[9] S.-G. Zhou, PoS INPC2016, 373 (2017).

[10] M. Freer, H. Horiuchi, Y. Kanada-En'yo, D. Lee, and U.-G. Meissner, Rev. Mod. Phys. 90, 035004 (2018).

[11] T. Otsuka, A. Gade, O. Sorlin, T. Suzuki, and Y. Utsuno, Rev. Mod. Phys. 92, 015002 (2020).

[12] I. Tanihata, H. Hamagaki, O. Hashimoto, Y. Shida, N. Yoshikawa, K. Sugimoto, O. Yamakawa, T. Kobayashi, and N. Takahashi, Phys. Rev. Lett.
55, 2676 (1985).

[13] P. G. Hansen and B. Jonson, Europhys. Lett. 4, 409 (1987).

[14] J. Dobaczewski, W. Nazarewicz, T. R. Werner, J. F. Berger, C. R. Chinn, and J. Dechargé, Phys. Rev. C 53, 2809 (1996).

[15] J. Meng and P. Ring, Phys. Rev. Lett. 77, 3963 (1996).

[16] J. Meng and P. Ring, Phys. Rev. Lett. 80, 460 (1998).

[17] J. Meng, Nucl. Phys. A 635, 3 (1998).

[18] A. S. Jensen, K. Riisager, D. V. Fedorov, and E. Garrido, Rev. Mod. Phys. 76, 215 (2004).

[19] K. Riisager, Phys. Scr. T152, 014001 (2013).

[20] T. Misu, W. Nazarewicz, and S. Ȧberg, Nucl. Phys. A 614, 44 (1997).

[21] S.-G. Zhou, J. Meng, P. Ring, and E.-G. Zhao, Phys. Rev. C 82, 011301(R) (2010).

[22] T. Nakamura, N. Kobayashi, Y. Kondo, Y. Satou, N. Aoi, H. Baba, S. Deguchi, N. Fukuda, J. Gibelin, N. Inabe, M. Ishihara, D. Kameda, Y. Kawada, T. Kubo, K. Kusaka, A. Mengoni, T. Motobayashi, T. Ohnishi, M. Ohtake, N. A. Orr, H. Otsu, T. Otsuka, A. Saito, H. Sakurai, S. Shimoura, T. Sumikama, H. Takeda, E. Takeshita, M. Takechi, S. Takeuchi, K. Tanaka, K. N. Tanaka, N. Tanaka, Y. Togano, Y. Utsuno, K. Yoneda, A. Yoshida, and K. Yoshida, Phys. Rev. Lett. 103, 262501 (2009).

[23] T. Nakamura, N. Kobayashi, Y. Kondo, Y. Satou, J. A. Tostevin, Y. Utsuno, N. Aoi, H. Baba, N. Fukuda, J. Gibelin, N. Inabe, M. Ishihara, D. Kameda, T. Kubo, T. Motobayashi, T. Ohnishi, N. A. Orr, H. Otsu, T. Otsuka, H. Sakurai, T. Sumikama, H. Takeda, E. Takeshita, M. Takechi, S. Takeuchi, Y. Togano, and K. Yoneda, Phys. Rev. Lett. 112, 142501 (2014).

[24] N. Kobayashi, T. Nakamura, Y. Kondo, J. A. Tostevin, Y. Utsuno, N. Aoi, H. Baba, R. Barthelemy, M. A. Famiano, N. Fukuda, N. Inabe, M. Ishihara, R. Kanungo, S. Kim, T. Kubo, G. S. Lee, H. S. Lee, M. Matsushita, T. Motobayashi, T. Ohnishi, N. A. Orr, H. Otsu, T. Otsuka, T. Sako, H. Sakurai, Y. Satou, T. Sumikama, H. Takeda, S. Takeuchi, R. Tanaka, Y. Togano, and K. Yoneda, Phys. Rev. Lett. 112, 242501 (2014).

[25] W. H. Long, P. Ring, J. Meng, N. Van Giai, and C. A. Bertulani, Phys. Rev. C 81, 031302 (2010).

[26] I. Hamamoto, Phys. Rev. C 85, 064329 (2012).

[27] K. Fossez, W. Nazarewicz, Y. Jaganathen, N. Michel, and M. Płoszajczak, Phys. Rev. C 93, 011305(R) (2016).

[28] K. Fossez, J. Rotureau, N. Michel, Q. Liu, and W. Nazarewicz, Phys. Rev. C 94, 054302 (2016).

[29] X.-X. Sun, J. Zhao, and S.-G. Zhou, Phys. Lett. B 785, 530 (2018).

[30] L. Fortunato, J. Casal, W. Horiuchi, J. Singh, and A. Vitturi, Commun. Phys. 3, 132 (2020).

[31] K. Yoshida, Phys. Rev. C 102, 054336 (2020).

[32] C. W. Johnson, K. D. Launey, N. Auerbach, S. Bacca, B. R. Barrett, C. R. Brune, M. A. Caprio, P. Descouvemont, W. H. Dickhoff, C. Elster, P. J. Fasano, K. Fossez, H. Hergert, M. Hjorth-Jensen, L. Hlophe, B. Hu, R. M. I. Betan, A. Idini, S. König, K. Kravvaris, D. Lee, J. Lei, A. Mercenne, R. N. Perez, W. Nazarewicz, F. M. Nunes, M. Płoszajczak, J. Rotureau, G. Rupak, A. M. Shirokov, I. Thompson, J. P. Vary, A. Volya, F. Xu, R. G. T. Zegers, V. Zelevinsky, and X. Zhang, J. Phys. G: Nucl. Part. Phys. 47, 123001 (2020). 
[33] L.-L. Li, J. Meng, P. Ring, E.-G. Zhao, and S.-G. Zhou, Phys. Rev. C 85, 024312 (2012).

[34] L.-L. Li, J. Meng, P. Ring, E.-G. Zhao, and S.-G. Zhou, Chin. Phys. Lett. 29, 042101 (2012).

[35] X.-X. Sun, J. Zhao, and S.-G. Zhou, Nucl. Phys. A 1003, $122011(2020)$.

[36] J. C. Pei, Y. N. Zhang, and F. R. Xu, Phys. Rev. C 87, 051302(R) (2013).

[37] Y. Chen, P. Ring, and J. Meng, Phys. Rev. C 89, 014312 (2014).

[38] H. Nakada and K. Takayama, Phys. Rev. C 98, 011301(R) (2018).

[39] I. Hamamoto, Phys. Rev. C 95, 044325 (2017).

[40] J. L. Egido, Phys. Scr. 91, 073003 (2016).

[41] L. M. Robledo, T. R. Rodríguez, and R. R. RodríguezGuzmán, J. Phys. G: Nucl. Part. Phys. 46, 013001 (2019).

[42] T. R. Rodríguez and J. L. Egido, Phys. Lett. B 705, 255 (2011).

[43] Z. P. Li, T. Nikšić, and D. Vretenar, J. Phys. G: Nucl. Part. Phys. 43, 024005 (2016).

[44] M. Bender, P. Bonche, and P.-H. Heenen, Phys. Rev. C 74, 024312 (2006).

[45] R. R. Rodríguez-Guzmán, J. L. Egido, and L. M. Robledo, Phys. Rev. C 69, 054319 (2004).

[46] T. Nikšić, D. Vretenar, G. A. Lalazissis, and P. Ring, Phys. Rev. Lett. 99, 092502 (2007).

[47] T. R. Rodríguez and J. L. Egido, Phys. Lett. B 663, 49 (2008).

[48] T. R. Rodríguez and J. L. Egido, Phys. Rev. Lett. 99, 062501 (2007).

[49] M. Bender and P.-H. Heenen, Phys. Rev. C 78, 024309 (2008).

[50] T. R. Rodríguez and J. L. Egido, Phys. Rev. C 81, 064323 (2010).

[51] J. M. Yao, J. Meng, P. Ring, and D. P. Arteaga, Phys. Rev. C 79, 044312 (2009).

[52] J. M. Yao, J. Meng, P. Ring, and D. Vretenar, Phys. Rev. C 81, 044311 (2010).

[53] J. M. Yao, K. Hagino, Z. P. Li, J. Meng, and P. Ring, Phys. Rev. C 89, 054306 (2014).

[54] J. L. Egido, M. Borrajo, and T. R. Rodríguez, Phys. Rev. Lett. 116, 052502 (2016).

[55] F.-Q. Chen and J. L. Egido, Phys. Rev. C 95, 024307 (2017).

[56] F. Q. Chen, Q. B. Chen, Y. A. Luo, J. Meng, and S. Q. Zhang, Phys. Rev. C 96, 051303(R) (2017).

[57] F. Chen, J. Meng, and S. Zhang, Phys. Lett. B 785, 211 (2018).

[58] P. Marević and N. Schunck, Phys. Rev. Lett. 125, 102504 (2020).

[59] J. L. Egido and A. Jungclaus, Phys. Rev. Lett. 125, 192504 (2020).

[60] S.-G. Zhou, J. Meng, and P. Ring, Phys. Rev. C 68, 034323 (2003).

[61] Y. Chen, L. Li, H. Liang, and J. Meng, Phys. Rev. C 85, 067301 (2012).

[62] K. Y. Zhang, D. Y. Wang, and S. Q. Zhang, Phys. Rev. C 100, 034312 (2019).

[63] C. Pan, K. Zhang, and S. Zhang, Int. J. Mod. Phys. E 28, 1950082 (2019).

[64] K. Zhang, M.-K. Cheoun, Y.-B. Choi, P. S. Chong, J. Dong, L. Geng, E. Ha, X. He, C. Heo, M. C. Ho, E. J. In, S. Kim, Y. Kim, C.-H. Lee, J. Lee,
Z. Li, T. Luo, J. Meng, M.-H. Mun, Z. Niu, C. Pan, P. Papakonstantinou, X. Shang, C. Shen, G. Shen, W. Sun, X.-X. Sun, C. K. Tam, Thaivayongnou, C. Wang, S. H. Wong, X. Xia, Y. Yan, R. W.-Y. Yeung, T. C. Yiu, S. Zhang, W. Zhang, and S.-G. Zhou, Phys. Rev. C 102, 024314 (2020).

[65] E. J. In, P. Papakonstantinou, Y. Kim, and S.-W. Hong, Int. J. Mod. Phys. E , 2150009 (2021).

[66] Z. H. Yang, Y. Kubota, A. Corsi, K. Yoshida, X.X. Sun, J. G. Li, M. Kimura, N. Michel, K. Ogata, C. X. Yuan, Q. Yuan, G. Authelet, H. Baba, C. Caesar, D. Calvet, A. Delbart, M. Dozono, J. Feng, F. Flavigny, J.-M. Gheller, J. Gibelin, A. Giganon, A. Gillibert, K. Hasegawa, T. Isobe, Y. Kanaya, S. Kawakami, D. Kim, Y. Kiyokawa, M. Kobayashi, N. Kobayashi, T. Kobayashi, Y. Kondo, Z. Korkulu, S. Koyama, V. Lapoux, Y. Maeda, F. M. Marqués, T. Motobayashi, T. Miyazaki, T. Nakamura, N. Nakatsuka, Y. Nishio, A. Obertelli, A. Ohkura, N. A. Orr, S. Ota, H. Otsu, T. Ozaki, V. Panin, S. Paschalis, E. C. Pollacco, S. Reichert, J.-Y. Roussé, A. T. Saito, S. Sakaguchi, M. Sako, C. Santamaria, M. Sasano, H. Sato, M. Shikata, Y. Shimizu, Y. Shindo, L. Stuhl, T. Sumikama, Y. L. Sun, M. Tabata, Y. Togano, J. Tsubota, F. R. Xu, J. Yasuda, K. Yoneda, J. Zenihiro, S.-G. Zhou, W. Zuo, and T. Uesaka, Phys. Rev. Lett. 126, 082501 (2021).

[67] P. Ring and P. Schuck, The Nuclear Many-Body Problem (Springer-Verlag Berlin Heidelberg, 1980).

[68] K. Hara and Y. Sun, Int. J. Mod. Phys. E 4, 637 (1995).

[69] M. Bender, P. Bonche, T. Duguet, and P.-H. Heenen, Phys. Rev. C 69, 064303 (2004).

[70] R. Rodríguez-Guzmán, J. L. Egido, and L. M. Robledo, Nucl. Phys. A 709, 201 (2002).

[71] J. Yao, H. Mei, and Z. Li, Phys. Lett. B 723, 459 (2013).

[72] J. M. Yao, M. Bender, and P.-H. Heenen, Phys. Rev. C 91, 024301 (2015).

[73] T. R. Rodríguez, A. Poves, and F. Nowacki, Phys. Rev. C 93, 054316 (2016).

[74] P. W. Zhao, Z. P. Li, J. M. Yao, and J. Meng, Phys. Rev. C 82, 054319 (2010).

[75] T. Baumann, A. M. Amthor, D. Bazin, B. A. Brown, C. M. F. III, A. Gade, T. N. Ginter, M. Hausmann, M. Matoš, D. J. Morrissey, M. Portillo, A. Schiller, B. M. Sherrill, A. Stolz, O. B. Tarasov, and M. Thoennessen, Nature 449, 1022 (2007).

[76] H. L. Crawford, P. Fallon, A. O. Macchiavelli, P. Doornenbal, N. Aoi, F. Browne, C. M. Campbell, S. Chen, R. M. Clark, M. L. Cortés, M. Cromaz, E. Ideguchi, M. D. Jones, R. Kanungo, M. MacCormick, S. Momiyama, I. Murray, M. Niikura, S. Paschalis, M. Petri, H. Sakurai, M. Salathe, P. Schrock, D. Steppenbeck, S. Takeuchi, Y. K. Tanaka, R. Taniuchi, H. Wang, and K. Wimmer, Phys. Rev. Lett. 122, 052501 (2019).

[77] N. Tsunoda, T. Otsuka, K. Takayanagi, N. Shimizu, T. Suzuki, Y. Utsuno, S. Yoshida, and H. Ueno, Nature 587, 66 (2020).

[78] S. R. Stroberg, J. D. Holt, A. Schwenk, and J. Simonis, Phys. Rev. Lett. 126, 022501 (2021).

[79] J. Erler, N. Birge, M. Kortelainen, W. Nazarewicz, E. Olsen, A. M. Perhac, and M. Stoitsov, Nature 486, 509 (2012).

[80] Q. Z. Chai, J. C. Pei, N. Fei, and D. W. Guan, Phys. Rev. C 102, 014312 (2020). 
[81] J. M. Yao, H. Mei, H. Chen, J. Meng, P. Ring, and D. Vretenar, Phys. Rev. C 83, 014308 (2011).

[82] X.-Y. Wu and X.-R. Zhou, Phys. Rev. C 92, 054321 (2015).
[83] T. Bürvenich, D. G. Madland, J. A. Maruhn, and P.-G. Reinhard, Phys. Rev. C 65, 044308 (2002).

[84] T. R. Rodríguez, Eur. Phys. J. A 52, 190 (2016).

[85] E. Caurier, F. Nowacki, and A. Poves, Phys. Rev. C 90, 014302 (2014). 